\title{
ULK1 Gene
}

National Cancer Institute

\section{Source}

National Cancer Institute. ULK1 Gene. NCI Thesaurus. Code C116612.

This gene is involved in the regulation of autophagy. 